\title{
Exploring the Fine-Layer Structure Around a Glissonean Pedicle in Cadaveric Models
}

\author{
Mamoru Morimoto, Yoichi Matsuo, Goro Ueda, Tomokatsu Kato, Yoshinaga Aoyama, Yuichi Hayashi, \\ Kan Omi, Hiroyuki Imafuji, Kenta Saito, Ken Tsuboi, Ryo Ogawa, Hiroki Takahashi, Shuji Takiguchi
}

Department of Gastroenterological Surgery,

Nagoya City University Graduate School of Medical Sciences, Japan

\section{ABSTRACT}

Background: Glissonean pedicle isolation is a very useful procedure during a laparoscopic anatomic hepatectomy (LAH); however, few studies have reported the precise layer structure around a Glissonean pedicle. The aim of this study was to evaluate the layer structure around a Glissonean pedicle in cadaveric models and determine whether Glissonean pedicle isolation based on the layer structure can serve as a standard surgical procedure during a $\mathrm{LAH}$.

Methods: From April 2017 to December 2019, LAHs were performed in 59 patients. Prior to the LAH, a cadaveric model was used to verify the layer structure around the Glissonean pedicle. The procedure was also performed in live patients during LAHs and pathologic verification was performed. In addition, we evaluated the short-term results of LAHs.

Results: Laennec's capsule covering the liver parenchyma was shown by histologic examination (Elastica van Gieson staining) in a cadaveric model and live patients. The gap between the Glissonean pedicle and Laennec's capsule was dissected without parenchymal destruction. A LAH based on cadaver simulation had an operative time of $398 \mathrm{~min}$ and blood loss of $142 \mathrm{ml}$. Post-operative complications occurred in 2 patients (3\%), and there were no mortalities. The length of hospital stay was 13 days.

Conclusion: There is a layer structure around the Glissonean pedicle; it is necessary to recognize Laennec's capsule as a landmark. When performing Glissonean pedicle isolation, it is possible to perform a safer and more precise LAH by placing importance on Laennec's capsule.

Key words: Glissonean pedicle isolation, cadaveric model, laparoscopic anatomic hepatectomy

\section{INTRODUCTION}

Laparoscopic surgery has been standardized in Japan and the safety and efficacy of laparoscopic surgery has been evaluated (1). Specifically, laparoscopic hepatectomy has increased in popularity due to minimal invasiveness (2-4). Recently, the efficacy of an anatomic hepatectomy for hepatocellular carcinoma (HCC) has been reported (5). Moreover, a nonanatomic tissue-sparing hepatec-

\author{
Corresponding author: \\ Yoichi Matsuo, M.D., Ph.D. \\ Department of Gastroenterological \\ Surgery, Nagoya City University Graduate \\ School of Medical Science \\ Kawasumi 1, Mizuho-cho, Mizuhoku \\ Nagoya 467-8601, Japan \\ Tel: +81-52-853-8226 \\ Fax: $+81-52-842-3906$ \\ E-mail: matsuo@med.nagoya-cu.ac.jp
}

Received: 18.02 .2020

Accepted: 26.03 .2020 
tomy is associated with worse disease-free survival (DFS) in patients with KRAS-mutated tumors. Therefore, more extensive anatomic hepatectomy for KRASmutated tumors is warranted (6). Glissonean pedicle isolation while performing an anatomic hepatectomy is a simple and useful procedure, and Glissonean pedicle isolation is a widely performed procedure during open surgery $(7,8)$; however, the procedure is difficult to perform during laparoscopic surgery because the risk of vascular injury may be increased due to motion restriction. Sugioka et al. (9) reported that it is important to understand the structure of Laennec's capsule when performing Glissonean pedicle isolation.

Therefore, it is necessary to understand the layer structure around the Glissonean pedicle in greater detail. There is a limit, however, to acquiring anatomic knowledge in a live patient. Traditionally embalmed cadavers are generally not suitable due to denaturation of the tissues. Soft-fix embalmed cadavers, such as those embalmed using the Thiel method, are used to train advanced surgical operations and because living tissue texture and color are preserved (10). Can Glissonean pedicle isolation be safely performed during laparoscopic anatomic hepatectomy (LAH) in live patients after simulation with Thiel-embalmed cadavers?

The purpose of this study was to determine whether Glissonean pedicle isolation in the appropriate layer as a landmark contributes to a safe and reliable LAH. To achieve this purpose, we examined the Glissonean pedicle isolated in the appropriate layer pathologically in cadaveric models. If an appropriate layer between the Glissonean pedicle and liver parenchyma can be clarified, even less experienced surgeons will be able to perform a safe LAH by dissecting the Glissonean pedicle while viewing the appropriate layer directly.

\section{METHODS}

\section{Cadaver simulation}

From April to September 2016, three Thielembalmed cadavers were used for this study. The cadavers were donated to the Department of Anatomy of Nagoya City University. Before the patients died, the patients signed documents agreeing to donate their body for use in clinical studies. The format of the document meets the criteria for the Japanese "Act on Body Donation for Medical and Dental Education." All cadavers were embalmed using the method described by Thiel (10). The cadavers were embalmed in a water-based solution consisting of salt with a small amount of formaldehyde for fixation, boric acid for disinfection, glycol, chlorocresol, and ethanol; this precipitation results in tissue homogenization. The skin is life-like and the joints are fully flexible. To prove the existence of Laennec's capsule, the specimens with separated Glissonean pedicles in cadaveric models were Elastica van Gieson-stained and inspected.

\section{Patients}

This study included patients who underwent surgical resection of solitary liver tumors based on preoperative radiologic images from April 2017 to December 2019 after cadaver simulation. A total of 59 patients underwent LAHs at Nagoya City University. All operations were performed by one experienced laparoscopic surgeon (M.M.) who obtained endoscopic surgeon qualifications from the Japan Society for Endoscopic Surgery. Clinical characteristics, such as intra- and post-operative data, were retrospectively obtained from medical records.

\section{RESULTS}

\section{Concept of dissecting the Glissonean pedicle (figure 1, videos 1, videos 2)}

Laennec's capsule covers not only the entire surface of the liver parenchyma beneath the serosa, including the bare area, but also the intrahepatic parenchyma surrounding the Glissonean pedicles and the plate system (figure 1a). At first glance, the serosa and Laennec's capsule appear to be the same membrane; however, these membranes are not the same. After the serosa is divided, a clear boundary between these membranes can be confirmed (figure 1 b). Videos 1 and 2 shown right and left Glissonean pedicle isolation in cadaveric models, respectively.

\section{Macroscopic findings (figure 2)}

First, we divided the serosa covering the right Glissonean pedicle and liver parenchyma in a cadaveric model [figure 2a (black arrow)]. Then, the gap between the anterior Glissonean pedicle and a thin membrane on the liver parenchyma was separated (figure $2 b$ ).

The right side of the left Glissonean pedicle (figure 2c) was separated from the left side of the left Glissonean pedicle in the dorsal direction (figure 2d). Laennec's capsule was clearly noted as a thin membrane during laparoscopy. 

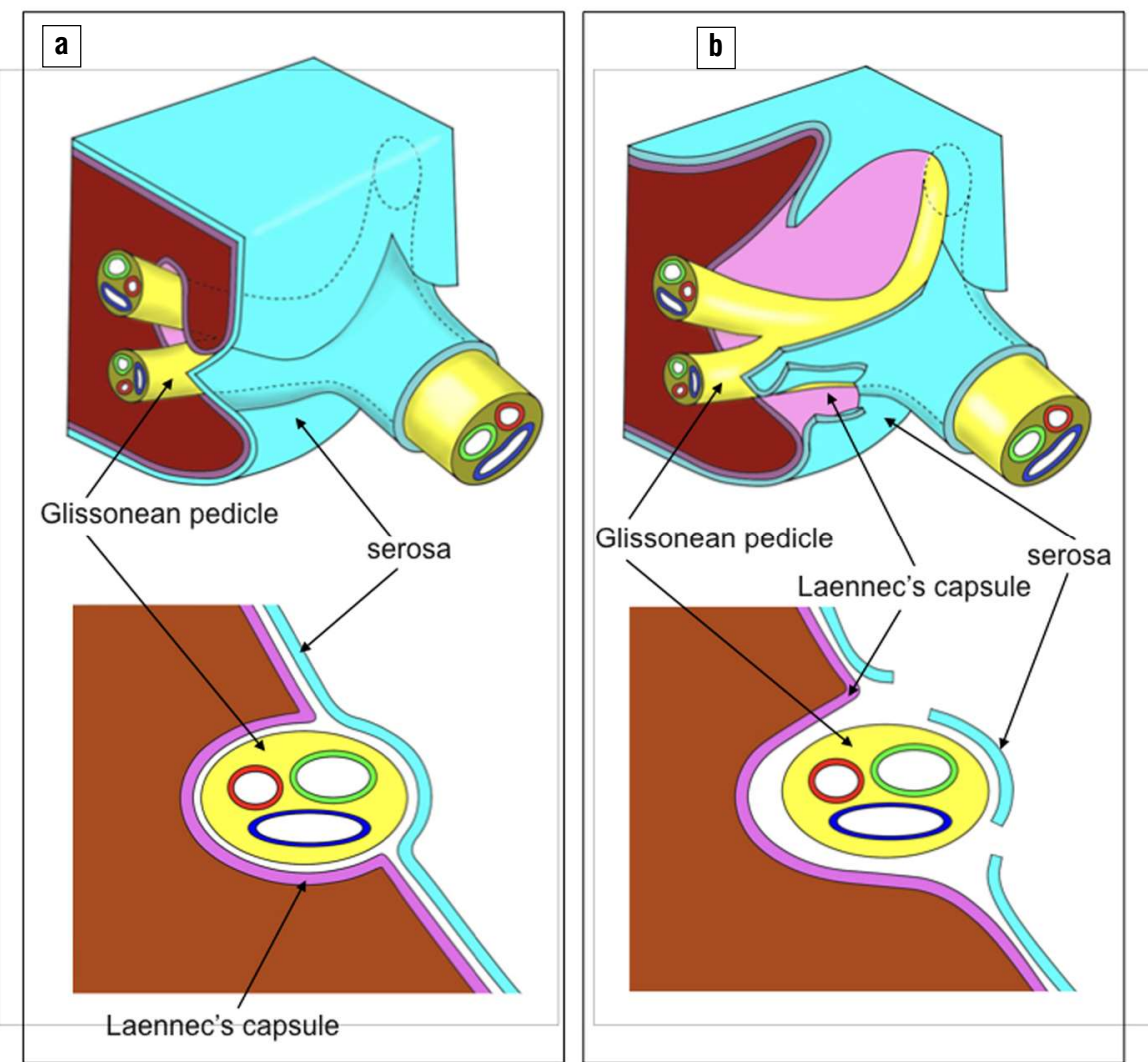

Figure 1 - Concept of dissecting the Glissonean pedicle isolation. Laennec's capsule covers not only the entire surface of the liver parenchyma beneath the serosa but also the intrahepatic parenchyma surrounding the Glissonean pedicles

(a). After the serosa is divided, a gap between the Glissonean pedicles and Laennec's capsule can be confirmed and separated clearly (b).
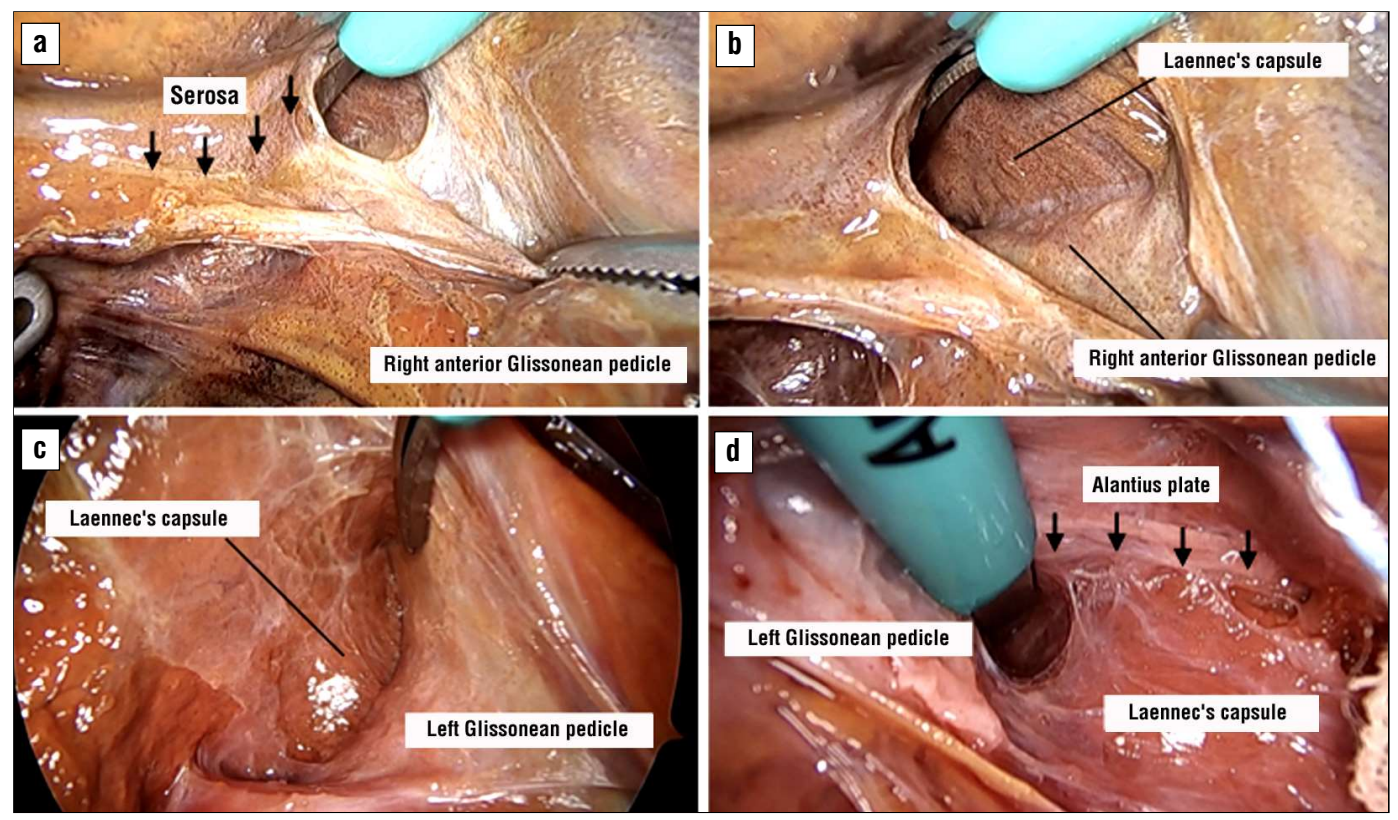

Figure 2 - Glissonean pedicle isolation focused on layer structure in cadaveric models.

Right Glissonean pedicle isolation: First, we cut the serosa covering the anterior

Glissonean pedicle and liver parenchyma in a cadaveric model

(a) After that, the gap between Laennec's capsule and the anterior Glissonean pedicle was separated (b). Left Glissonean pedicle isolation: The separation of the gap between Laennec's capsule and the right side of the left Glissonean pedicle (c), the left side of the left Glissonean pedicle in the dorsal direction. (d). Laennec's capsule is clearly recognized as a thin membrane. 


\section{Histologic findings (figure 3)}

We also attempted to elucidate the layer structure around the Glissonean pedicle histologically using Elastica van Gieson staining. We confirmed the existence of a membrane on the liver parenchyma in cadaveric models. The location of the specimen for pathological examination is shown (figure $3 a$ ). A dense fibrous layer (black arrows) was observed on the liver parenchyma during right Glissonean pedicle isolation (figure $3 b$ (low-power field) and figure $3 c$ (high-power field)). These membranes were Laennec's capsule. The gap between the Glissonean pedicle and Laennec's capsule was exfoliated without parenchymal destruction.

\section{Patient and tumor characteristics (table 1)}

Table 1 shows patient details, including age, gender, body mass index (BMI), indications for surgery, tumor location, and the size of the tumor.

\section{Operative and postoperative data (table 2)}

Table 2 demonstrates the operative and postoperative patient data. Two of 59 patients (3\%) required blood transfusions. The mean operative time was 398 min (range, 291-578 min). The mean blood loss was 142 $\mathrm{mL}$ (range, 82-372 $\mathrm{mL}$ ). Postoperative complications occurred in $3 \%$ of the patients (2/59). Bile leakage was noted in two patients and deemed to be grade B according to the International Study Group of Liver Surgery (ISGLS) classification for bile leakage (11). No

Table 1 - Patient's and tumor characteristics

\begin{tabular}{lc}
\hline & LAH (n=59) \\
\hline Age (uears) & $67(37-79)$ \\
\hline Gender & 34 \\
Male & 25 \\
Female & \\
$\ldots$ BMI indications & 35 \\
HCC & 6 \\
CCC & 18 \\
CRLM & \\
Tumor location & 2 \\
Segment I & 3 \\
Segment II & 9 \\
Segment III & 8 \\
Segment IV & 8 \\
Segment V & 10 \\
Segment VI & 7 \\
Segment VII & 12 \\
Segment VIII & $34(19-45)$ \\
\hline Size of tumor (mm)
\end{tabular}

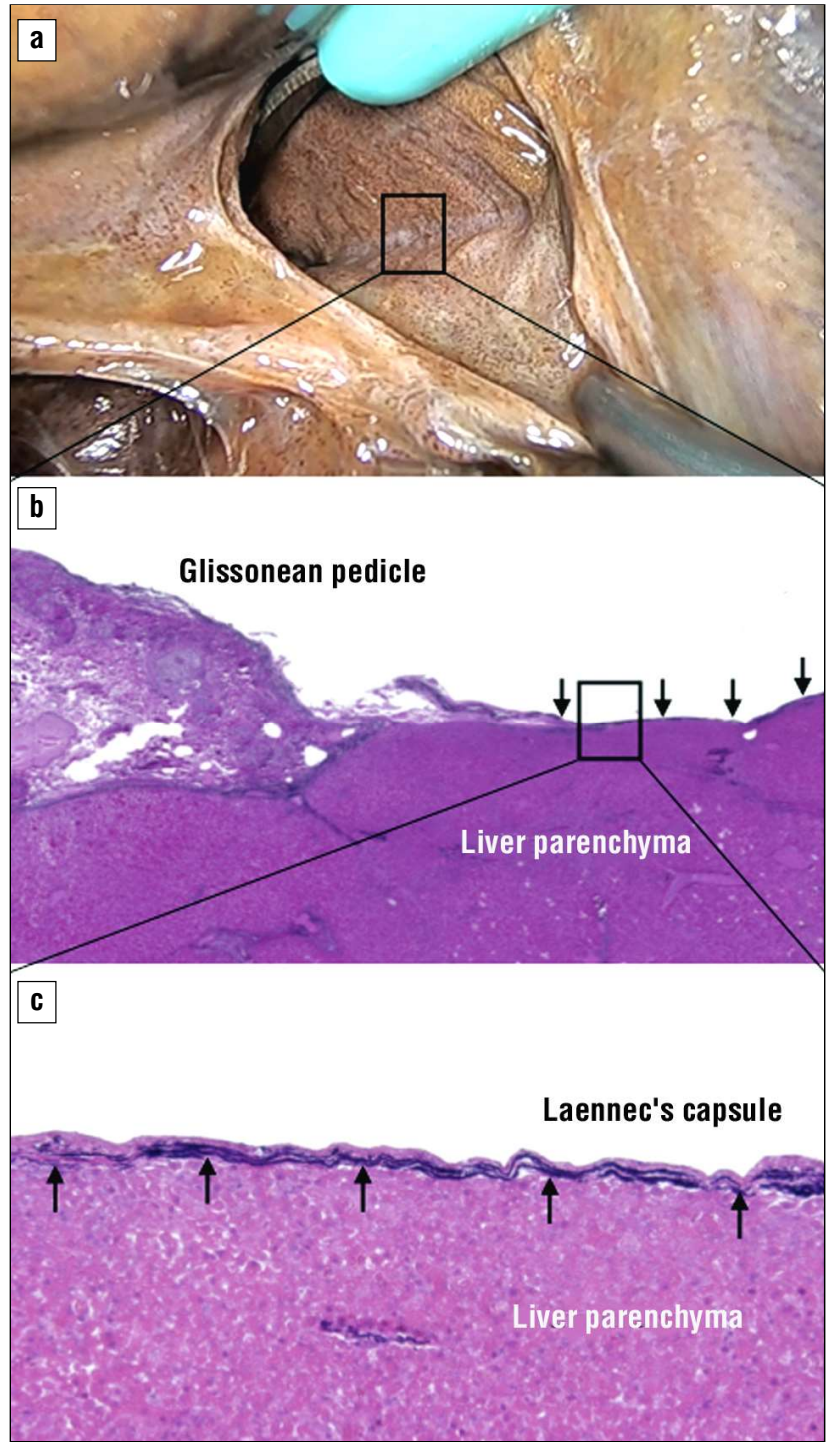

Figure 3 - Histologic findings of Laennec's capsule in cadaveric models (Elastica van Gieson staining).

Right Glissonean pedicle isolation: The location of the specimen for pathological examination is shown (a). Laennec's capsule on the liver parenchyma and exfoliated right anterior Glissonean pedicle of the cadaveric model is shown in a low-power field (b), and high-power field (c). A dense fibrous layer (black arrows) was observed on the liver parenchyma; these membranes were Laennec's capsule. It was shown that the gap between the Glissonean pedicle and Laennec's capsule can be exfoliated without parenchymal destruction.

perioperative mortalities occurred. The mean length of hospital stay was 13 days (range, 7-32 days).

\section{DISCUSSION}

The detailed layer structure around the Glissonean pedicle (particularly Laennec's capsule) was pathologically verified in cadavers. The operative results of LAH with extrahepatic Glissonean pedicle isolation with a focus on Laennec's capsule was shown to be safe and feasible. We are of the opinion that extrahepatic Glissonean pedicle isolation with a focus on 
Table 2 - Operative and postoperative data

\begin{tabular}{lc}
\hline & LAH (n=59) \\
\hline Type of resection & \\
Subsegmentectomy & 21 \\
Anterior subsegmentectomy & 5 \\
Posterior subsegmentectomy & 7 \\
Central bisegmentectomy & 2 \\
Left lobectomy & 13 \\
Right lobectomy & 11 \\
\hline Blood transfusion & $2(3 \%)$ \\
\hline Operative time (min) & $398(291-578)$ \\
Blood loss (ml) & $142(82-372)$ \\
\hline Postoperative complications & $2(3 \%)$ \\
Bile leakage & $2(3 \%)$ \\
Postoperative mortality & 0 \\
\hdashline Length of hospital stay (day) & $13(7-32)$ \\
\hline
\end{tabular}

Laennec's capsule is an important and essential technique in safely performing a LAH.

The Glissonean pedicle isolation technique is a useful method during anatomic hepatectomy and is a widely used technique. The Glissonean pedicle isolation technique has been reported to be effective for shortening the operative time, reducing the amount of bleeding, and reducing the complication rate (12-14); however, the Glissonean pedicle isolation technique is not a standard procedure during LAH. During laparoscopic hepatectomy it is difficult to operate because of motion restrictions and there are few landmarks to isolate the Glissonean pedicle. Thus, it is necessary to understand the layer structure as a clear landmark for standardization.

Many discussions have involved the layer structure around the intrahepatic vessels. Walaeus (15) first described the vasculo-biliary sheath, which contains the portal vein, the hepatic artery, and the bile duct, in 1640. Glisson (16) also reported the Glissonean pedicle in 1642. In 1802, Laennec (17) first described a proper membrane as the distinct structure from the serosa. Couinaud established the concept of the plate system as a fibrous thickening part of the Glissonean sheath (18) and demonstrated that Laennec's capsule has no continuity with the Glissonean pedicle (19). Hayashi et al. (20) conducted a precise histologic study of cadaveric livers with elastic fiber and lymphatic vein staining and demonstrated that the so-called Glissonean capsule was not derived from the Glissonean sheath, but from Laennec's capsule surrounding the pedicles, and that Laennec's capsule extended to the peripheral Glissonean pedicles. Sugioka et al. (9) first reported that Laennec's capsule served as a landmark to isolate the extrahepatic Glissonean pedicle. A gap exists between the Glissonean pedicle and Laennec's capsule, and it is possible to isolate the extrahepatic Glissonean pedicle without parenchymal destruction.

Simulation of advanced surgical operations in traditionally embalmed cadavers is often not possible due to the rigidity of the tissues; however, Thiel cadavers may be suitable for training of a broad range of surgical technical skills (21). Thiel embalming fluid contains formaldehyde in a very low concentration, along with glycol, water, and various salts, and hence is safer than a traditional embalming medium (22). Following the embalming process, the tissue is preserved without the need for refrigeration or special storage facilities. Previous reports have highlighted the potential advantages of this technique for certain surgical specialties (23-26).

In this study we used a thiel embalming cadaver model to simulate the Glissonean pedicle isolation technique with a focus on the detailed layer structure, especially Laennec's capsule. The specimen was removed and the layer structure was verified pathologically to confirm the presence of Laennec's capsule on liver parenchyma clearly. Furthermore, it was shown that Glissonean pedicle isolation with a focus on Laennec's capsule may contribute to good operative results during LAH. Indeed, Laennec's capsule is an important structure and landmark for Glissonean pedicle isolation during LAH.

\section{CONCLUSION}

In the future, minimal invasive surgery, such as laparoscopic and robotic surgery, will become commonplace during liver surgery. However, anatomical knowledge has never been changed. To that end, it is necessary to recognize the fine layer structure facilitated by the magnified effect of a laparoscope and Laennec's capsule can be an important landmark in extrahepatic Glissonean pedicle isolation.

\section{Conflict of interest}

All author declare that they have no conflict of interest.

\section{REFERENCES}

1. Inomata M, Shiroshita H, Uchida H, Bandoh T, Akira S, Yamaguchi S, et al. Current status of endoscopic surgery in Japan: The 14th National Survey of Endoscopic Surgery by the Japan Society for Endoscopic Surgery. Asian J Endosc Surg. 2020;13(1):7-18. Epub 2019 Dec 11.

2. Nguyen KT, Gamblin TC, Geller DA. World review of laparoscopic liver resection-2,804 patients. Ann Surg. 2009; 250: 831-41. 
3. Otsuka Y, Tsuchiya M, Maeda T, Katagiri T, Isii J, Tamura A, Yamazak K, Kubota Y, Suzuki T, Kagami S, Kaneko H. Laparoscopic hepatectomy for liver tumors: proposals for standardization. J Hepatobiliary Pancreat Surg. 2009; 16:720-725.

4. Hwang DW, Han HS, Yoon YS, Cho JY, Kwon Y, Kim JH, et al Laparoscopic major liver resection in Korea: a multicenter study. J Hepatobiliary Pancreat Sci. 2013; 20: 125-130.

5. Huang X, Lu S. A Meta-analysis comparing the effect of anatomical resection vs. non-anatomical resection on the long-term outcomes for patients undergoing hepatic re-section for hepatocellular carcinoma. HPB(Oxford) 2017; 19: 843-849.

6. Margonis GA, Buettner S, Andreatos N, Sasaki K, ljzermans JNM, van Vugt JLA, et al. Anatomical resections improve disease-free survival in patients with KRAS-mutated colorectal liver metastases. Ann Surg. 2017;266(4):641-649.

7. Couinaud CM. A simplified method for controlled left hepatectomy. Surgery 1985; 97: 358-361.

8. Takasaki K, Kobayashi S, Tanaka S. Newly developed systematized hepatectomy by Glissonean pedicle transection method. Shujutsu 1986; 40: 7-14.

9. Sugioka A, Kato Y, Tanahashi Y. Systematic extrahepatic Glissonean pedicle isolation for anatomical liver resection based on Laennec's capsule: proposal of a novel comprehensive surgical anatomy of the liver. J Hepatobiliary Pancreat Sci 2017; 24: 17-23.

10. Thiel W. The preservation of the whole corpse with natural color. Ann Anat 1992; 174: 185-195.

11. Koch M, Garden OJ, Padbury R, Rahbari NN, Adam R, Capussotti L et al : Bile leak- age after hepatobiliary and pancreatic surgery : a definition and grading of severity by the International Study Group of Liver Surgery. Surgery. 2011; 149(5):680-8. Epub 2011 Feb 12.

12. Lee N, Cho CW, Kim JM, Choi GS, Kwon CHD, Joh JW1. Application of temporary inflow control of the Glissonean pedicle method provides a safe and easy technique for totally laparoscopic hemihepatectomy by Glissonean approach. Ann Surg Treat Res. 2017; 92(5):383-386. Epub 2017 Apr 27.

13. Hu Y, Shi J, Wang S, Zhang W, Sun X, Sun B, et al. Laennec's approach for laparoscopic anatomic hepatectomy based on Laennec's capsule. BMC Gastroenterol. 2019:19(1):194.

14. Eguchi S, Kanematsu T, Arii S, Okazaki M, Okita K, Omata M, et al. Comparison of the outcomes between an anatomical subsegmen- tectomy and a non-anatomical minor hepatectomy for single hepatocellular carcinomas based on a Japanese nation- wide survey. Surgery. 2008; 143: 469-475.

15. Walaeus J. Epistolae duae de motu chyli et sanguinis ad Thomam Bartholeum. Leiden: Fransciscus Hackius; 1640.

16. Glisson F. Anatomia hepatis. London: O. Pullein; 1642.

17. Laennec RTH. Lettre sur des Tuniques qui enveloppent certains Visceres,et fournis-sentdes gaines membraneuses a leurs vaisseaux. Journ De Med Chir et Pharm Ven-demiaire an Xl; p.539-575, et Germinal an XI; 1802. p.73-89.

18. Couinaud C. Liver lobes and segments: notes on the anatomical architecture and surgery of the liver. Presse Med. 1954; 62: 709-712.

19. Couinaud C. The vasculo-biliary sheath. Surgical anatomy of the liver revisited. Paris: pers Ed; 1989: 29-39.

20. Hayashi S, Murakami G, Ohtsuka A, Itoh M, Nakano T, Fukuzawa Y. Connective tissue configuration in the human liver hilar region with special reference to the liver capsule and vascular sheath. J HepatoBiliary-Pancreat Surg. 2008; 15: 640-7.

21. Yiasemidou M, Roberts D, Glassman D, Tomlinson J, Biyani S, Miskovic S. A multi-specialty evaluation of Thiel cadavers for surgical training. World J Surg. 2017; 41: 1201-1207.

22. Eisma R, Mahendran S, Majumdar S, Smith D, Soames RW. A comparison of Thiel and formalin embalmed cadavers for thyroid surgery training. Surgeon. 2011;9(3):142-6. Epub 2010 Oct 6.

23. Holzle F, Franz EP, Lehmbrock J, Weihe S, Teistra C, Deppe H, et al. Thiel embalming technique: a valuable method for teaching oral surgery and implantology. Clin Implant Dent Relat Res. 2012; 14(1):121-6. Epub 2009 Aug 6.

24. Peuker ET, Werkmeister R, Pera F, Joos U, Filler TJ. Surgical procedures in mouth, jaw and facial surgery in Thiel embalmed body donors. Mund Kiefer Gesichtschir. 2001;5(2):141-3. German

25. Giger U, Frésard I, Häfliger A, Bergmann $M$, Krähenbühl $L$. Laparoscopic training on Thiel human cadavers: a model to teach advanced laparoscopic procedures. Surg Endosc. 2008;22(4):9016. Epub 2007 Aug 18.

26. Wolff KD, Kesting M, Mücke T, Rau A, Hölzle F. Thiel embalming technique: a valuable method for microvascular exercise and teaching of flap raising. Microsurgery. 2008;28(4):273-8. 